\title{
No detectable genetic correlation between male and female mating frequency in the stalk-eyed fly Cyrtodiopsis dalmanni
}

\author{
CA Grant ${ }^{1}$, T Chapman ${ }^{2}$, A Pomiankowski ${ }^{1}$ and K Fowler ${ }^{1}$ \\ ${ }^{1}$ Department of Biology, University College London, Wolfson House, 4 Stephenson Way, London NW1 2HE, UK; ${ }^{2}$ Department of \\ Biology, University College London, Darwin Building, Gower Street, London WC1E 6BT, UK
}

\begin{abstract}
There is much interest in explaining why female insects mate multiply. Females of the stalk-eyed fly Cyrtodiopsis dalmanni can mate several times each day in a lifetime which may span several months. There are many adaptive explanations, but one hypothesis that has received little rigorous empirical attention is that female multiple mating has evolved for nonadaptive reasons as a correlated response to selection for high male mating frequency rather than because of direct or indirect benefits accruing to females. We tested this hypothesis in stalk-eyed flies by measuring the mating frequency of females from lines that exhibited a direct
\end{abstract}

response in males to artificial selection for increased ('high') and decreased ('low') male mating frequency. We found that the mating frequency of high-line females did not differ from that of low-line females. Hence, there was no support for a genetic correlation between male and female mating frequency in this species. Our study suggests that the genes which influence remating may not be the same in the sexes, and that females remate frequently in this species to gain as yet unidentified benefits.

Heredity (2005) 95, 444-448. doi:10.1038/sj.hdy.6800733; published online 10 August 2005

Keywords: genetic correlation; mating frequency; multiple mating; stalk-eyed fly

\section{Introduction}

In general, males are expected to have a higher optimal mating frequency than females (Arnqvist and Nilsson, 2000; Gavrilets et al, 2001). This is because males are assumed to be able to produce sperm in unlimited quantity and so increase their reproductive success with successive matings in contrast to the situation for females (Bateman, 1948). In females, reproductive traits such as egg production are assumed to be limiting, so each extra mating beyond an optimum value may provide diminishing benefits (Bateman, 1948; Parker, 1979). However, multiple mating is in fact common in female insects (Thornhill and Alcock, 1983; Ridley, 1988) and it is therefore important to evaluate the rival explanations for this phenomenon.

Females may benefit directly from multiple mating by obtaining sufficient sperm to fertilise available eggs (Ridley, 1988; Arnqvist and Nilsson, 2000). Also female fitness may be enhanced by the receipt of nutrients, for example, prey items (Thornhill and Alcock, 1983) which may increase fecundity (Boggs and Gilbert, 1979; Butlin et al, 1987). Female multiple mating may also yield indirect, genetic benefits. For example, genes enhancing offspring fitness can be acquired through the adaptive consequences of sperm competition (Curtsinger, 1991; Keller and Reeve, 1995; Yasui, 1997). Similarly, multiple mating may reduce genetic incompatibilities and the

Correspondence: K Fowler, Department of Biology, University College London, Wolfson House, 4 Stephenson Way, London NW1 2HE, UK; E-mail:k.fowler@ucl.ac.uk

Received 22 April 2005; accepted 8 July 2005; published online 10 August 2005 costs of inbreeding (eg Zeh and Zeh, 1997; Yasui, 1998; Tregenza and Wedell, 2002).

A contrasting and relatively unexplored nonadaptive hypothesis is that high female mating frequency has evolved due to a strong genetic correlation between the sexes in mating rate (Halliday and Arnold, 1987; Arnold and Halliday, 1988, 1992). If mating frequency is controlled by the same genes in males and females, then selection on males for higher remating would result in high remating frequency in females. This hypothesis assumes that selection on males to mate frequently is stronger than on females to mate at their lower optimal mating frequency (Halliday and Arnold, 1987).

The available data on whether there is a genetic correlation between the sexes in mating frequency are inadequate. Previous studies have used artificial selection experiments with Drosophila melanogaster (Manning, 1963; Gromko and Newport, 1988; Stamenkovic-Radak et al, 1992; Sgrò et al, 1998). However, genetic drift (Arnold and Halliday, 1988, 1992) or assortative mating (Butlin, 1993; see also Stamenkovic-Radak et al, 1993) are plausible explanations for the correlated responses reported in some of the Drosophila studies (Manning, 1963; Gromko and Newport, 1988; Stamenkovic-Radak et al, 1992). The most rigorously designed experiment shows no genetic correlation between the sexes (Sgrò et al, 1998). Taken together, these data provide mixed results and little evidence of a genetic correlation between the sexes for remating frequency in D. melanogaster. A major problem is that none of the previous studies practised artificial selection on mating frequency per se. Instead, they focused selection on the time to first mating (Manning, 1963; Stamenkovic-Radak et al, 1992) 
or on the length of time between the first and the second matings (Gromko and Newport, 1988; Sgrò et al, 1998). Genetic correlations between the sexes in related traits are of interest, but may be misleading since their sign and/or magnitude may differ markedly from that between male and female mating frequency itself. Hence, a rigorous test for the presence of a genetic correlation between the sexes directly on mating frequency rather than related traits is needed to evaluate the correlational hypothesis.

Bi-directional artificial selection for male mating frequency may be difficult to achieve logistically in species such as $D$. melanogaster which may mate typically only once every 1 or 2 days. This is because it is difficult to actually observe the required number of matings to impose selection and so traits related to mating frequency, such as time to first mating, have instead been the focus of selection in previous studies. However, the sexually dimorphic stalk-eyed fly, Cyrtodiopsis dalmanni presents an ideal opportunity to more readily practise selection on mating frequency, because mating rates are extremely high (Wilkinson and Dodson, 1997). In the laboratory, females can mate more than 10 times in a morning (Reguera et al, 2004). A second and important motivation for this study is that it is crucially important to examine the reasons for multiple mating in species other than $D$. melanogaster in order to establish generality. Stalk-eyed flies are of further interest when testing for the potential benefits of remating, because they represent a rare opportunity among insects to study determinants of female choice. They exhibit dramatic lateral extension of the head capsule with the eyes displaced onto the ends of stalks. In C. dalmanni, eyespan is greater in males than in females of comparable body size (Burkhardt and de la Motte, 1988) and females prefer to roost and mate with males bearing the largest eyespan (Wilkinson and Reillo, 1994; Hingle et al, 2001).

Here we test the hypothesis that the high frequency of female multiple mating in $C$. dalmanni results from a genetic correlation for mating frequency between the sexes. We used lines that had undergone eight generations of artificial selection for increased ('high' lines) and decreased ('low' lines) male mating frequency (Rogers et al, 2005) and showed direct responses in males to selection. The lines were founded from a wild-type base population exhibiting significant phenotypic and genetic variation in diverse morphological and life history traits in both sexes (David et al, 1998, 2000). We tested the genetic correlation between the sexes in mating frequency by making a direct measure of mating frequency (number of matings observed) for females from replicate high, low and control lines.

\section{Materials and methods}

\section{Fly stocks and culturing}

The base stock was a laboratory population founded in 1993 from individuals captured by AP in Gombak, Malaysia. The stock was maintained at high numbers in population cages (typically 10 cages with $>200$ individuals per cage). The cages (height $=20 \mathrm{~cm}$, width $=20 \mathrm{~cm}$, length $=30 \mathrm{~cm}$ ) had a base of damp cotton to maintain cage humidity, at an approximately 1:1 sex ratio. Flies were kept at $25^{\circ} \mathrm{C}$ on a $12: 12 \mathrm{~h}$ dark:light cycle with a 30-min period of reduced lighting to simulate dawn and dusk. Flies were fed puréed sweet corn, to which a mould inhibitor $(10 \% \mathrm{w} / \mathrm{v}$ Nipagin in $100 \%$ ethanol) was added. Flies for the experiment were obtained by collecting eggs from population cages on petri dishes and raising emerging larvae to adulthood. To obtain virgins, flies were segregated by sex 14-21 days after eclosion, using ice anaesthesia. Virgin flies were then placed in single sex population cages.

\section{Artificial selection lines}

Full details of the artificial selection protocol are given in Rogers et al (2005). In brief, two replicate lines were selected for increased male mating frequency ('high' selection regime), two replicate lines were selected for decreased male mating frequency ('low' selection regime) and there were two unselected 'control' lines. All lines were derived from the wild-type laboratory population described above. Mating frequency was measured as the total number of matings achieved in a 1.5-h period commencing at artificial dawn on each of two consecutive days. In each generation, the mating frequencies of 24 males from each selection line were assayed with base stock females. The eight males with the highest or lowest mating frequencies were mated to females of their own line to produce the next generation of the high or low lines, respectively. Within each control line, the mating frequency of 12 males was measured and eight males were randomly chosen. There was a direct response to selection for male mating frequency. High-line and low-line males mated significantly more and less frequently, respectively, than did control males (Rogers et al, 2005). For example, at generation 7 of selection: mean \pm SD for replicate one, high-mating males $=14.53 \pm 1.32$ matings, low-mating males $=8.98 \pm$ 0.43 ; for replicate two, high-mating males $=13.43 \pm 1.06$, low-mating males $=10.23 \pm 0.70$.

\section{Correlated response in female mating frequency}

Female mating frequency was assayed using females from the eighth generation of selection, when there was significant divergence between high and low lines in male mating frequency (Rogers et al, 2005). For the assay, we replicated the conditions under which male mating frequency had been assessed during artificial selection and provided females from the selection lines with unselected base stock males.

At 2 days prior to behavioural observations, sets of five females from each replicate line (1 or 2) of each selection regime (high, control or low) were placed in assay pots $(400 \mathrm{ml}$ : height $=95 \mathrm{~mm}$, diameter $=75 \mathrm{~mm})$ containing a damp cotton wool base and $2 \mathrm{~g}$ of puréed sweetcorn. On the morning of observation, a randomly chosen single male was added to each set of females. On each of three consecutive days, observation began at the start of artificial dawn and lasted $1.5 \mathrm{~h}$. The total number of matings over $40 \mathrm{~s}$ by each set of females ('number of matings') was recorded.

During the study, we obtained measures for a series of eight groups. Each group was comprised of four assay pots for each replicate line of each selection regime, yielding a total sample size over the entire experiment of 32 sets of females per line. To control for variation in male mating frequency within the 3-day assays, indivi- 
dual males were moved between selection line females after each consecutive day; one day with high-line females, one day with low-line females and one day with control-line females. The sequence of exposure to females from each selection regime was randomly assigned.

Statistical analysis was performed using JMP statistical software (version 5, SAS Institute Inc.) for the Apple Macintosh.

\section{Results}

The number of matings for females from each selection regime, defined as the total number of matings by all five females in each assay pot, are summarised in Table 1 . The number of matings was normally distributed (ShapiroWilk tests), while the experimental group did not have a significant effect on the number of matings (oneway ANOVA $F_{7,184}=0.87, P>0.5$ ). Therefore, data were pooled across experimental groups for further analysis.

The number of matings was analysed using a fully balanced nested ANOVA with selection regime as a fixed effect and replicate line nested within selection regime as a random effect (Table 2). There was no difference in the mean number of matings of females from the high, low or control selection regimes $\left(F_{2,3}=0.24, P>0.75\right)$. There were no significant differences between replicates within selection regimes $\left(F_{3,186}=1.05, P>0.25\right)$.

\section{Discussion}

There was no evidence for a genetic correlation between male and female mating frequency in the stalk-eyed fly C. dalmanni. Artificial selection for increased and decreased male mating frequency (number of matings) produced a direct response (Rogers et al, 2005). We found no significant differences in the mating frequency of females, estimated directly as the number of matings, from the high, low or control selection lines using the same assay conditions as those used to test male flies from these lines. Remating rates in our assays were lower than reported elsewhere (eg Reguera et al, 2004), possibly because the female biased ratio used in our assays could have reduced the mating opportunities for each female.

If mating frequency was controlled by the same genes in males and females, females might mate beyond their optimal frequency if there was stronger selection on males to mate at a high frequency than on females to mate just once or a few times (Halliday and Arnold, 1987). Strong selection on females to reduce the mating frequency could lead to the evolution of modifying

Table 1 Mean \pm standard error for the total number of matings observed in 1.5-h periods on 3 consecutive days, for sets of five females from the high, low and control lines artificially selected for male mating frequency

\begin{tabular}{lccc}
\hline $\begin{array}{l}\text { Selection } \\
\text { regime }\end{array}$ & Replicate & $\begin{array}{c}\text { Number of } \\
\text { matings }\end{array}$ & $\begin{array}{c}\text { Number of } \\
\text { sets assayed }\end{array}$ \\
\hline High & 1 & $13.22 \pm 0.78$ & 32 \\
High & 2 & $11.97 \pm 0.74$ & 32 \\
Control & 1 & $12.50 \pm 0.60$ & 32 \\
Control & 2 & $13.62 \pm 0.68$ & 32 \\
Low & 1 & $12.78 \pm 0.72$ & 32 \\
Low & 2 & $13.09 \pm 0.55$ & 32 \\
\hline
\end{tabular}

elements to limit the expression of genes which increase the mating frequency (Sherman and Westneat, 1988). The lack of genetic correlation between the sexes for mating frequency in C. dalmanni implies that mating frequency is under the control of different or sex-limited genes in males and females. Alternatively, it is possible that the selection pressures on mating frequency in males and female stalk-eyed flies are not divergent as generally predicted (Halliday and Arnold, 1987), and this is an interesting topic for future study. The potential existence of sex-limited genes determining the mating frequency in males and females suggests that constraints on selection on mating frequency in males and females may be minimised.

Relatively little is known about the determinants of female remating in C. dalmanni. Given a pairwise choice, females mate more frequently with males with large eyespan than those with small eyespan (Wilkinson et al, 1998; Hingle et al, 2001). Large females mate more than small females, while large females have significantly more mature eggs in their ovaries and may therefore mate at a higher frequency than small females to maintain high fertility (Grant, 2003). It is possible that the difference in mating frequency between large and small eyespan females reflects a male preference for large, fecund females. However, previous studies found no significant differences between selection regimes in eyespan, a measure highly correlated with body size (Rogers et al, 2005) or female fecundity (Segar et al, unpublished data). Remating in male and female $C$. dalmanni could be influenced by other factors, including environmental influences via physiological limitations such as ejaculate size or sperm storage capacity, availability of oviposition sites or chemical cues such as male accessory gland proteins. Future evaluation of the selection pressures on female mating frequency will need to assess the strength of the potential costs to remating in females (Reguera et al, 2004) relative to the likely benefits such as sperm replenishment.

The lack of a genetic correlation between the sexes for mating frequency in C. dalmanni is consistent with evidence from D. melanogaster (Sgrò et al, 1998). The factors involved in determining remating in D. melanogaster are well characterised and differ between the sexes. Female $D$. melanogaster are stimulated to remate by nutritional status (Harshman et al, 1988), availability of oviposition sites (Trevitt et al, 1988), the number of sperm in storage (Letsinger and Gromko, 1985) and accessory gland proteins transferred by mating males (Chen et al, 1988; Wolfner, 1997, 2002; Chapman, 2001; Chapman et al, 2003; Liu and Kubli, 2003). Males are stimulated to

Table 2 ANOVA of number of matings of females from the high, low and control lines artificially selected for male mating frequency, with selection regime (fixed effect) and replicate line nested within selection regime (random effect)

\begin{tabular}{lrrrr}
\hline Source of variation & df & MS & F & P \\
\hline Selection regime & 2 & 3.77 & 0.24 & $>0.75$ \\
Replicate line within selection regime $^{\mathrm{a}}$ & 3 & 15.60 & 1.05 & $>0.25$ \\
Error $^{\mathrm{b}}$ & 186 & 14.83 & & \\
\hline
\end{tabular}

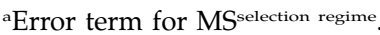

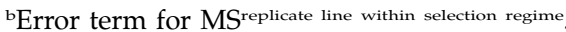


remate by visual stimuli (Willmund and Ewing, 1982), female cuticular hydrocarbons (Scott, 1986) and the number of recent matings obtained (Markow et al, 1978). If, as these findings suggest, different factors influence mating in males and females, it is unlikely that selection on the mating frequency in one sex will significantly affect the mating frequency of the unselected sex.

If the ancestral population for the selection lines exhibited low levels of genetic variation, then a genetic correlation between the sexes in $C$. dalmanni might be underestimated. However, the base population is maintained at relatively large population size and shows significant genetic variation in a variety of traits (including eyespan, thorax length and wing size parameters) in both sexes (David et al, 1998, 2000). There is no indication therefore that adaptation to the laboratory environment has removed genetic variance in general or genetic variance for female mating frequency in particular.

A genetic correlation between the sexes might also be obscured if there was inadvertent selection on female mating frequency within the lines (Butlin, 1993), for example, disassortative mating, where male $C$. dalmanni from 'high' lines preferentially mate with females with low mating frequency. Ideally, individual males and females should be paired randomly (eg Sgrò et al, 1998). Here, this was partially achieved by placing selected males each generation with five randomly chosen selection line females (Rogers et al, 2005). The imposition of equal family size can also reduce variation between lines due to disassortative mating (Sgrò et al, 1998). The procedure for these lines minimised, but did not eliminate, variation in family size. The contribution of selected males was equalised across regimes by taking an equal number of progeny from each male every generation, but it remains possible that the contribution by females varied across regimes.

The present study provided no evidence for a genetic correlation between males and females for mating frequency in the stalk-eyed fly, C. dalmanni. The hypothesis that female mating frequency has evolved as a correlated response to selection on male mating frequency is not supported. Our experiments therefore suggest that the high rate of female remating may be adaptive in this species and future work should focus on identifying potential explanations.

\section{Acknowledgements}

We thank NERC (studentship to CAG and research grant to TC, KF and AP), UCL (laboratory facilities) and the Royal Society (URF to TC) for funding. We thank the editor and three anonymous reviewers for helpful comments.

\section{References}

Arnold SJ, Halliday T (1988). Multiple mating: natural selection is not evolution. Anim Behav 36: 1547-1548.

Arnold SJ, Halliday T (1992). Multiple mating by females: the design and interpretation of selection experiments. Anim Behav 43: 178-179.
Arnqvist G, Nilsson T (2000). The evolution of polyandry: multiple mating and female fitness in insects. Anim Behav 60: 145-164.

Bateman AJ (1948). Intra-sexual selection in Drosophila. Heredity 2: 349-368.

Boggs CL, Gilbert LE (1979). Male contribution to egg production in butterflies: evidence for transfer of nutrients at mating. Science 206: 83-84.

Burkhardt D, de la Motte I (1988). Big 'antlers' are favoured: female choice in stalk-eyed flies (Diptera, Insecta), field collected harems and laboratory experiments. J Comp Physiol A 162: 649-652.

Butlin RK (1993). A comment on the evidence for a genetic correlation between the sexes in Drosophila melanogaster. Anim Behav 45: 403-404.

Butlin RK, Woodhatch CW, Hewitt GM (1987). Male spermatophore investment increases female fecundity in a grasshopper. Evolution 41: 221-225.

Chapman T (2001). Seminal fluid-mediated fitness traits in Drosophila. Heredity 87: 511-521.

Chapman T, Bangham J, Vinti G, Seifried B, Lung O, Wolfner MF et al (2003). The sex peptide of Drosophila melanogaster: female post-mating responses analysed by using RNA interference. Proc Natl Acad Sci USA 100: 9923-9928.

Chen PS, Stumm-Zollinger E, Aigaki T, Balmer J, Bienz M, Böhlen P (1988). A male accessory gland peptide that regulates reproductive behaviour of female $D$. melanogaster. Cell 54: 291-298.

Curtsinger JW (1991). Sperm competition and the evolution of multiple mating. Am Nat 138: 93-102.

David P, Bjorksten T, Fowler K, Pomiankowski A (2000). Condition-dependent signalling of genetic variation in stalk-eyed flies. Nature 406: 186-188.

David P, Hingle A, Greig D, Rutherford A, Pomiankowski A Fowler K (1998). Male sexual ornament size but not asymmetry reflects condition in stalk-eyed flies. Proc $R$ Soc Lond B 265: 2211-2216.

Gavrilets S, Arnqvist G, Friberg U (2001). The evolution of female mate choice by sexual conflict. Proc $R$ Soc Lond $B \mathbf{2 6 8}$ : 531-539.

Grant CA (2003). The evolution of multiple mating in the stalk-eyed fly, Cyrtodiopsis dalmanni. PhD Thesis, University College London.

Gromko MH, Newport MEA (1988). Genetic basis for remating in Drosophila melanogaster II. Response to selection based on the behaviour of one sex. Behav Genet 18: 621-632.

Halliday T, Arnold SJ (1987). Multiple mating by females: a perspective from quantitative genetics. Anim Behav 35: 939-941.

Harshman LG, Hoffmann AA, Prout T (1988). Environmental effects on remating in Drosophila melanogaster. Evolution 42: 312-321.

Hingle A, Fowler K, Pomiankowski A (2001). Size-dependent mate preference in the stalk-eyed fly, Cyrtodiopsis dalmanni. Anim Behav 61: 589-595.

Keller L, Reeve HK (1995). Why do females mate with multiple males? The sexually selected sperm hypothesis. Adv Study Behav 24: 291-315.

Letsinger JT, Gromko MH (1985). The role of sperm numbers in sperm competition and female remating in Drosophila melanogaster. Genetics 66: 195-202.

Liu H, Kubli E (2003). Sex peptide is the molecular basis of the sperm effect in Drosophila melanogaster. Proc Natl Acad Sci USA 100: 9929-9933.

Manning A (1963). Selection for mating speed in Drosophila melanogaster based on the behaviour of one sex. Anim Behav 11: $116-120$.

Markow TA, Quaid M, Kerr S (1978). Male mating experience and competitive courtship success in Drosophila melanogaster. Nature 276: 821-822. 
Parker GA (1979). Sexual selection and sexual conflict. In: Blum MS, Blum NA (eds) Sexual Selection and Reproductive Competition in Insects. Academic Press: New York, USA. pp 123-166.

Reguera P, Pomiankowski A, Fowler K, Chapman T (2004). Low cost of reproduction in female stalk-eyed flies, Cyrtodiopsis dalmanni. J Insect Physiol 50: 103-108.

Ridley M (1988). Mating frequency and fecundity in insects. Biol Rev 63: 509-549.

Rogers DW, Baker RH, Chapman T, Denniff M, Pomiankowski A, Fowler K (2005). Direct and correlated responses to artificial selection on male mating frequency in the stalk-eyed fly, Cyrtodiopsis dalmanni. J Evol Biol 18: 642-650.

Scott D (1986). Sexual mimicry regulates the attractiveness of mated Drosophila melanogaster females. Proc Natl Acad Sci USA 83: 8429-8433.

Sgrò CM, Chapman T, Partridge L (1998). Sex-specific selection on time to remate in Drosophila melanogaster. Anim Behav 56: 1267-1278.

Sherman PW, Westneat DF (1988). Multiple mating and quantitative genetics. Anim Behav 36: 1545-1547.

Stamenkovic-Radak M, Partridge L, Andjelkovic M (1992). A genetic correlation between the sexes for mating speed in Drosophila melanogaster. Anim Behav 43: 389-396.

Stamenkovic-Radak M, Partridge L, Andjelkovic M (1993). Genetic correlation between the sexes in Drosophila melanogaster: a reply to Butlin. Anim Behav 45: 405.

Thornhill R, Alcock J (1983). The Evolution of Insect Mating Systems. Harvard University Press: Cambridge, MA, USA.
Tregenza T, Wedell N (2002). Polyandrous females avoid costs of inbreeding. Nature 415: 71-73.

Trevitt S, Fowler K, Partridge L (1988). An effect of egg deposition on the subsequent fertility and remating frequency of female Drosophila melanogaster. J Insect Physiol 34: 821-828.

Wilkinson G, Dodson G (1997). Function and evolution of antlers and eye stalks in flies. In: Choe JC, Crespi BJ (eds) The Evolution of Mating Systems in Insects and Arachnids. Cambridge University Press: Cambridge, UK. pp 273-293.

Wilkinson GS, Kahler H, Baker RH (1998). Evolution of female mating preference in stalk-eyed flies. Behav Ecol 9: 525-533.

Wilkinson GS, Reillo PR (1994). Female choice response to artificial selection on an exaggerated male trait in a stalkeyed fly. Proc R Soc Lond B 255: 1-6.

Willmund R, Ewing A (1982). Visual signals in the courtship of Drosophila melanogaster. Anim Behav 30: 209-215.

Wolfner MF (1997). Tokens of love: functions and regulation of Drosophila male accessory gland products. Insect Biochem Mol Biol 27: 179-192.

Wolfner MF (2002). The gifts that keep on giving: physiological functions and evolutionary dynamics of male seminal proteins in Drosophila. Heredity 88: 85-93.

Yasui Y (1997). A 'good-sperm' model can explain the evolution of costly multiple mating by females. Am Nat 149: 573-584.

Yasui Y (1998). The 'genetic benefits' of female multiple mating reconsidered. Trends Ecol Evol 13: 246-250.

Zeh JA, Zeh DW (1997). The evolution of polyandry II: postcopulatory defenses against genetic incompatibility. Proc $R$ Soc Lond B 264: 69-75. 